\title{
Producción hidropónica de tomate (Solanum lycopersicum L.) en cascarilla de arroz mezclada con materiales minerales y orgánicos
}

\section{Soilless tomato production (Solanum lycopersicum L.) in rice hulls mixed with mineral and organic materials}

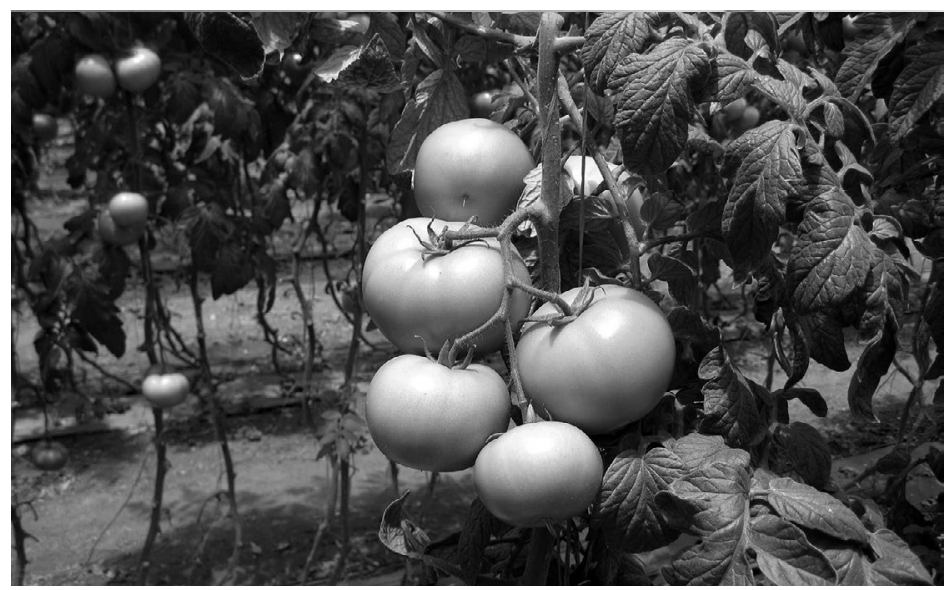

MAYDA Y. PEÑA' 1

FÁNOR CASIERRA-POSADA 2,4

OSCAR I. MONSALVE ${ }^{3}$

Tomates producidos bajo

invernadero en

Sutamarchán (Boyacá).

Foto: F. Casierra-Posada

\section{RESUMEN}

La existencia de factores limitantes del suelo, como salinización, agotamiento de la fertilidad natural y deterioro físico, conlleva a una búsqueda del mejoramiento de las tecnologías utilizadas para el desarrollo y la producción del tomate. Se llevó a cabo un ensayo en Chía (Colombia), en las instalaciones del Centro de Bio-Sistemas de la Universidad de Bogotá Jorge Tadeo Lozano donde se determinó el efecto de diferentes sustratos acondicionados a partir de materiales orgánicos e inertes sobre la producción y calidad del fruto en tomate bajo condiciones de invernadero. Se utilizó el híbrido Vitoria del que se seleccionaron frutos de acuerdo a su categoría comercial (primera, segunda, tercera e industrial). Los materiales utilizados para la preparación de los sustratos fueron: cascarilla cruda, cascarilla quemada, cascarilla cielo abierto, escoria de carbón, fibra de coco y zeolita, evaluados física y químicamente. Como parámetros de respuesta se tomaron: sólidos solubles totales, acidez titulable, $\mathrm{pH}$ y pérdida de peso. La acidez total titulable y los sólidos solubles totales aumentaron dependiendo del sustrato en el cual fueron sembrados donde la zeolita en mezcla con la cascarilla quemada incrementó los sólidos solubles totales y en mezcla con cascarilla cielo abierto incrementó la acidez titulable. Los valores de $\mathrm{pH}$ no presentaron diferencias respecto a los sustratos. La mayor producción de calidad primera se obtuvo con las plantas sembradas en zeolita en mezcla con cascarilla quemada seguida de este mismo material en mezcla con cascarilla cielo abierto.

\footnotetext{
Palabras clave adicionales: sustratos, sólidos solubles totales, acidez total titulable, pH, pérdida de peso.

Har-Best Colombia S.A.S., Tunja (Colombia).

2 Facultad de Ciencias Agropecuarias, Grupo de Investigación Ecofisiología Vegetal, Universidad Pedagógica y Tecnológica de Colombia, Tunja (Colombia).

Centro de Bio-Sistemas, Universidad de Bogotá Jorge Tadeo Lozano, Chía (Colombia).

4 Autor para correspondencia: fanor.casierra@uptc.edu.co
} 


\section{ABSTRACT}

Limiting factors of soil, such as salinization, depletion of natural fertility and physical deterioration, have led to the search for improved technologies for use in the development and production of the tomato. A study was carried out in Chia (Colombia) at the Bio-Systems Center of the Jorge Tadeo Lozano University that aimed to determine the effect of different substrates based on organic and inert materials on the production and fruit quality of tomatoes grown under greenhouse conditions. The hybrid Vitoria was used as the plant material and the fruits were selected according to their commercial classification (first, second, third and industrial). The materials used for the preparation of substrates were: raw rice hulls, burned rice hulls, open-air rice hulls, slag coal, coconut fiber and zeolite, which were evaluated physically and chemically. The response parameters were: total soluble solids, total titratable acidity, $\mathrm{pH}$ and weight loss. The total titratable acidity and total soluble solids increased depending on the substrate used. Zeolite mixed with burned rice hulls induced an increase in the total soluble solids and, when mixed with open-air rice hulls, increased acidity. The $\mathrm{pH}$ of the fruit juice values did not differ with respect to the substrates. The highest production quality was obtained with plants grown in a mixture of zeolite and burned rice hulls and of zeolite and openair rice hulls.

Additional key words: substrates, total soluble solids, total titratable acidity, pH, weight loss.
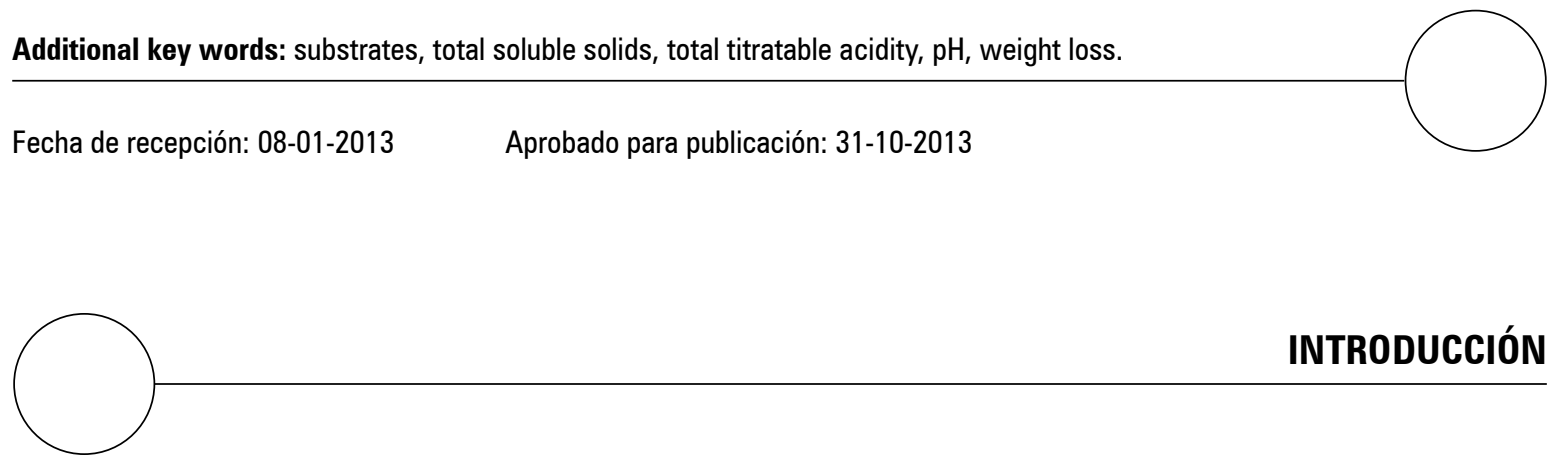

El tomate (Solanum lycopersicum L.) es uno de los cultivos donde se ha encontrado una mayor aceptación y más rápida difusión del cultivo sin suelo (Cánovas, 1995). Es uno de los cultivos más importantes del mundo. En Colombia se reportó una producción de 513.900 t en 2009 , con una tasa de crecimiento promedio anual de $2,5 \%$ en la producción y de $3,7 \%$ en el rendimiento (Agronet, 2010). El consumo per cápita de tomate a nivel mundial se calcula en $17 \mathrm{~kg}$ al año, mientras que en Colombia se estima en $9,34 \mathrm{~kg}$ al año. El tomate es uno de los productos que forma parte de la apuesta exportadora colombiana, por las enormes expectativas de producción y por los grandes potenciales de exportación (Presidencia de la República de Colombia, 2007).

Entre los problemas que se han observado en la producción hortícola a campo abierto, está la dificultad en el manejo de los factores que influyen en el desarrollo del cultivo como los cambios extremos de temperatura, la deficiencia o exceso de agua y los suelos mal drenados (Rodríguez et al., 2001). La hidroponía es una técnica para el desarrollo del cultivo en el que su sistema radical se desarrolla sin suelo, ya sea en agua o en sustratos inertes, con la particularidad que se debe proporcionar al sistema radical, agua, minerales y oxígeno suficientes para el óptimo desarrollo de la planta (Bastida, 2002). La combinación y proporción de los materiales del sustrato debe ser cuidadosamente estudiada, según los requerimientos de cada especie, pues el volumen limitado de los contenedores exige óptimas propiedades físicas y químicas para el crecimiento (Lavado, 2000).

La cascarilla de arroz es el material más empleado para los cultivos hidropónicos en Colombia bien sea cruda o parcialmente carbonizada. El principal inconveniente que presenta la cascarilla de arroz es su baja capacidad de retención 
de humedad y lo difícil que es lograr el reparto homogéneo de la misma (Calderón, 2002). La cascarilla cruda son los residuos de la cáscara o pajilla, que constituye aproximadamente $20 \%$ en peso del grano y que es separado del de pilado, la cascarilla quemada es el resultado de la cascarilla cruda quemada en un horno parcialmente y la cascarilla "cielo abierto" es la cascarilla cruda quemada a campo abierto.

Las zeolitas son minerales del grupo de los aluminosilicatos hidratados, con estructura porosa (porosidad mayor de 40\%), que presentan alta capacidad de retención de humedad $(25 \%$ p/p) y de intercambio catiónico (Qian et al., 2001). La fibra de coco está constituida por partes iguales de lignina y celulosa, es rica en potasio y en micronutrientes como Fe, Mn, Zn y Cu (Savithri et al., 1993). Las escorias son residuos de la quema de carbón mineral provenientes de hornos y calderas, muy utilizados por floricultores y viveristas para el enraizamiento (Calderón y Cevallos, 2001).

A pesar de que en Colombia el uso de sustratos ha logrado gran aceptación en plantaciones hortícolas, las investigaciones que involucran el uso de sustratos son escasas o reportan hallazgos que no permiten la toma de decisiones a partir de información confiable. Por otro lado, los sustratos diferentes al suelo ofrecen un gran número de ventajas, de las cuales, muchas no han sido suficientemente evaluadas en cultivos de importancia bajo condiciones de campo o invernadero. Por estas razones, el objetivo del presente estudio fue la evaluación de diferentes sustratos en rela- ción con la producción y calidad de tomate, bajo condiciones de invernadero.

\section{MATERIALES Y METODOS}

El trabajo se llevó a cabo en el invernadero Richel localizado en las instalaciones del Centro de Bio-Sistemas de la Universidad de Bogotá Jorge Tadeo Lozano, en el municipio de Chía (Cundinamarca), ubicado a $2.560 \mathrm{msnm}$. Durante el periodo del ensayo se registró en el invernadero una temperatura de $25 \pm 12^{\circ} \mathrm{C}$ y una humedad relativa de $42-90 \%$.

Se utilizó el híbrido Vitoria (Syngenta ${ }^{\circledR}$ ) como material vegetal. Los materiales utilizados para la preparación de los sustratos fueron cascarilla cruda, cascarilla quemada, cascarilla cielo abierto, escoria de carbón, fibra de coco comercial y zeolita, donde los tratamientos fueron: control o cascarilla cruda, cascarilla quemada en mezcla, con escoria de carbón, fibra de coco y zeolita , y cascarilla cielo abierto con escoria, fibra de coco y zeolita (tabla 1).

En el estudio se determinaron las características fisicoquímicas de los sustratos en mezcla. Se le realizaron pruebas físicas como porcentaje de humedad y materia seca, porcentaje de densidad aparente y densidad real, porcentaje de porosidad de aire, porcentaje retención de humedad y porcentaje de porosidad total. También se realizaron pruebas químicas como: $\mathrm{pH}, \mathrm{CE}, \mathrm{N}_{-} \mathrm{NH}_{4}$, $\mathrm{N}-\mathrm{NO}_{3}, \mathrm{~N}$ total, $\mathrm{P}, \mathrm{K}$ y CIC.

Tabla 1. Listado de combinaciones de materiales utilizados como sustrato en el ensayo.

\begin{tabular}{|c|l|}
\hline \multicolumn{1}{|c|}{$\begin{array}{c}\text { Fuente de variación } \\
\text { CE } 100 \%\end{array}$} & Testigo absoluto (cascarilla entera al 100\%) \\
\hline CO 70\%-EC 30\% & $70 \%$ cascarilla quemada $+30 \%$ escoria de carbón \\
\hline CO 70\%-FC 30\% & $70 \%$ cascarilla quemada $+30 \%$ fibra de coco \\
\hline CO 70\%-Ze 30\% & $70 \%$ cascarilla quemada $+30 \%$ zeolita \\
\hline CA 70\%-EC 30\% & $70 \%$ cascarilla cielo abierto $+30 \%$ escoria de carbón \\
\hline CA 70\%-FC 30\% & $70 \%$ cascarilla cielo abierto $+30 \%$ fibra de coco \\
\hline CA 70\%-Ze 30\% & $70 \%$ cascarilla cielo abierto $+30 \%$ zeolita \\
\hline
\end{tabular}


A los frutos se le realizaran pruebas físicas como peso fresco, peso seco, sólidos solubles totales, $\mathrm{pH}$ y acidez titulable, con ayuda de un refractómetro y un medidor de pH. Para determinar el efecto de los diferentes sustratos acondicionados a los parámetros de calidad y productividad del tomate, desde la primera cosecha se tomaron datos de peso total y calidad de fruto para cada una de las unidades experimentales (26 plantas). Para dicho fin se pesaron los frutos recolectados por cada cosecha y se tomaron datos de calidad comercial: primera $(72-81 \mathrm{~mm})$, segunda (62$71 \mathrm{~mm})$, tercera $(53-61 \mathrm{~mm})$ e industrial (46-52 $\mathrm{mm})$; cabe anotar que en el tomate industrial o calibre P incluye el tomate con cierto grado de malformaciones, leves rayones mecánicos o manchas de poco tamaño.

Se realizó un análisis clásico de variancia (Anava) y se aplicó la diferencia entre promedios mediante la prueba de Tukey $(P \leq 0,05)$. Los análisis estadísticos se realizaron con la versión 11.0 de SPSS. Las figuras se presentan en formato de barras con su respectiva desviación estándar.

\section{RESULTADOS Y DISCUSIÓN}

\section{Propiedades físicas de los sustratos}

Las diferentes mezclas de sustratos para sus propiedades físicas presentaron marcadas diferencias (tabla 2), especialmente debido al origen de los materiales (mineral o vegetal), observando que los resultados se presentaron opuestos para cada característica, donde, las mezclas realizadas con zeolita de origen mineral, contrarrestaron con las mezclas con fibra de coco de origen vegetal, las cuales representaron las tendencias más marcadas.

Según Cadahía (2005), normalmente, es difícil que un solo material reúna las características ideales para el desarrollo de los cultivos. Las propiedades físicas de los sustratos para el desarrollo de los cultivos en sustratos deben tener una alta capacidad de retención de agua, estar disponibles, tener suficiente aireación, alta estabilidad y homogeneidad, baja densidad aparente, alta porosidad total, y ser fácilmente mojable. Valdivia (1989) reportó que partículas inferiores a 0,25 $\mathrm{mm}$, tienen una gran influencia en la retención excesiva de humedad, creando problemas de aireación, además de que los sustratos que presentan una proporción por arriba del 15\% de este tamaño, se compactan demasiado. Verdonck et al. (1981) mencionan que para determinar el uso de un material como medio de cultivo o sustrato, uno de los factores más importantes a considerar son sus propiedades físicoquímicas, con la finalidad de programar, desde un inicio, el tipo de manejo a realizar, fundamentalmente en cuanto a nutrición, abastecimiento de agua, y todas aquellas circunstancias de manejo y uso. Wilson (1985) mencionó que el material fino incrementa la cantidad de agua fácilmente disponible, en tanto que el material grueso incrementa la capacidad de aireación.

\section{Propiedades químicas de los sustratos}

De los valores obtenidos en los materiales utilizados para el presente ensayo la zeolita presentó un alto valor de CIC (tabla 3), lo que concuerda con Handreck y Black (1991), quienes determinaron este material como el de mayor CIC comparado con otros materiales. La escoria de carbón y la cascarilla cielo abierto correspondieron a los valores más bajos (tabla 2). Según Ansorena (1994), aunque en ocasiones se emplean sustratos que apenas tienen CIC, generalmente es más sencillo el manejo cuando el medio presenta cierta capacidad de intercambio catiónico, ya que disminuyen los riesgos derivados de las pérdidas de nutrientes por lixiviación, a causa de los frecuentes e intensos riegos a los que se someten las plantas cultivadas en contenedor. Esta sería una ventaja para las plantas cultivadas en la mezcla realizada con zeolita ya que esta se caracteriza por su desempeño positivo en la capacidad de intercambio catiónico, el almacenamiento, la disponibilidad de nutrien- 


\begin{tabular}{|c|c|c|c|c|c|c|c|c|c|}
\hline \multirow[t]{2}{*}{ Fuente de variación } & Humedad & $\begin{array}{l}\text { Materia } \\
\text { seca }\end{array}$ & Cenizas & $\begin{array}{l}\text { Materia } \\
\text { orgánica }\end{array}$ & $\begin{array}{l}\text { Densidad } \\
\text { aparente }\end{array}$ & $\begin{array}{c}\text { Densidad } \\
\text { real }\end{array}$ & $\begin{array}{l}\text { Porosidad } \\
\text { de aire }\end{array}$ & \begin{tabular}{|c|} 
Capacidad \\
de \\
contenedor
\end{tabular} & $\begin{array}{c}\text { Porosidad } \\
\text { total }\end{array}$ \\
\hline & $(\%)$ & $(\%)$ & $(\%)$ & $(\%)$ & $\mathrm{g} \mathrm{cm}^{-3}$ & $\mathrm{~g} \mathrm{~cm}^{-3}$ & $(\%)$ & $(\%)$ & $(\%)$ \\
\hline $\begin{array}{l}\text { Cascarilla quemada } \\
+ \text { Fibra de coco }\end{array}$ & 61,19 & 38,81 & 16,42 & 83,58 & 0,15 & 1,62 & 33,12 & 67,45 & 90,73 \\
\hline $\begin{array}{l}\text { Cascarilla quemada } \\
+ \text { Zeolita }\end{array}$ & 32,83 & 74,69 & 68,53 & 31,47 & 0,54 & 2,13 & 33,61 & 23,31 & 74,65 \\
\hline $\begin{array}{l}\text { Cascarilla quemada } \\
+ \text { Escoria de } \\
\text { carbón }\end{array}$ & 44,27 & 55,73 & 43,75 & 56,25 & 0,29 & 1,86 & 35,29 & 53,14 & 84,69 \\
\hline $\begin{array}{l}\text { Cascarilla C. abierto } \\
+ \text { Fibra de coco }\end{array}$ & 47,32 & 52,68 & 26,26 & 80,80 & 0,18 & 1,64 & 27,20 & 82,38 & 88,92 \\
\hline $\begin{array}{l}\text { Cascarilla C. abierto } \\
+ \text { Zeolita }\end{array}$ & 31,68 & 68,32 & 61,61 & 47,79 & 0,30 & 1,95 & 25,94 & 40,80 & 84,97 \\
\hline $\begin{array}{l}\text { Cascarilla C. abierto } \\
+ \text { Escoria de } \\
\text { carbón }\end{array}$ & 42,16 & 57,84 & 76,23 & 32,06 & 0,29 & 2,26 & 7,32 & 57,62 & 87,16 \\
\hline
\end{tabular}

Tabla 3. Propiedades químicas de los sustratos usados en el ensayo.

\begin{tabular}{|c|c|c|c|c|c|c|c|c|c|c|}
\hline \multirow{2}{*}{$\begin{array}{l}\text { Fuente de } \\
\text { variación }\end{array}$} & CIC & $\mathrm{CO}$ & $\mathrm{pH}$ & CE & $N_{t}$ & $\mathrm{NH}_{4}^{+}$ & $\mathrm{NO}_{3}^{-}$ & $\mathrm{C} / \mathrm{N}$ & $\mathrm{P}$ & K \\
\hline & & $(\%)$ & & $\mathrm{dS} \mathrm{m}^{-1}$ & $(\%)$ & $(\%)$ & $(\%)$ & & $(\%)$ & $(\%)$ \\
\hline $\begin{array}{l}\text { Escoria de } \\
\text { carbón }\end{array}$ & 9,0 & 19,66 & 4,7 & 1,0 & 0,47 & 0,010 & 0,006 & 41,57 & 0,04 & 0,26 \\
\hline Zeolita & 60,7 & 1,88 & 5,8 & 0,3 & 0,02 & 0,003 & 0,003 & 82,14 & 0 & 0,42 \\
\hline Fibra de coco & 21,7 & 43,04 & 5,3 & 2,4 & 0,31 & 0,013 & 0,014 & 138,39 & 0,06 & 1,70 \\
\hline $\begin{array}{l}\text { Cascarilla } \\
\text { quemada }\end{array}$ & 14,1 & 34,17 & 5,9 & 1,6 & 0,36 & 0,010 & 0,015 & 95,72 & 0,05 & 0,44 \\
\hline $\begin{array}{l}\text { Cascarilla cielo } \\
\text { abierto }\end{array}$ & 8,3 & 32,02 & 6,3 & 1,7 & 0,55 & 0,011 & 0,009 & 58,75 & 0,11 & 0,54 \\
\hline
\end{tabular}

CIC: capacidad de intercambio catiónico; CO: carbono orgánico; CE: conductividad eléctrica; Nt: nitrógeno total; C/N: relación carbono / nitrógeno; P: fósforo; K: potasio.

tes, y la posibilidad de un mejor manejo de agua (Maloupa et al., 1992).

Características fisicoquímicas del tomate cultivado en las diferentes mezclas de sustratos

\section{Acidez total titulable (ATT)}

Respecto a este parámetro se encontraron diferencias altamente significativas $(P \leq 0,01)$ entre cascarillas así como en la interacción de cascarilla por sustrato. Se observó que la cascarilla cruda redujo los valores en esta variable en 11,2\% respecto a la cascarilla a cielo abierto (figura 1). El máximo valor de ATT se encontró en la mezcla cascarilla a cielo abierto junto con zeolita. Esta mezcla incrementó el valor de este parámetro en $16,7 \%$ respecto a la mezcla cascarilla quemada con zeolita.

Se presentaron valores entre 0,3 y $0,38 \%$ (figura 1) valores similares se encontraron en otros ensayos de tomate en sustratos donde se afirma que la calidad de tomate fue superior a la normal (Djedidi et al., 1997). Esto indica que los valores encontrados para el presente ensayo 


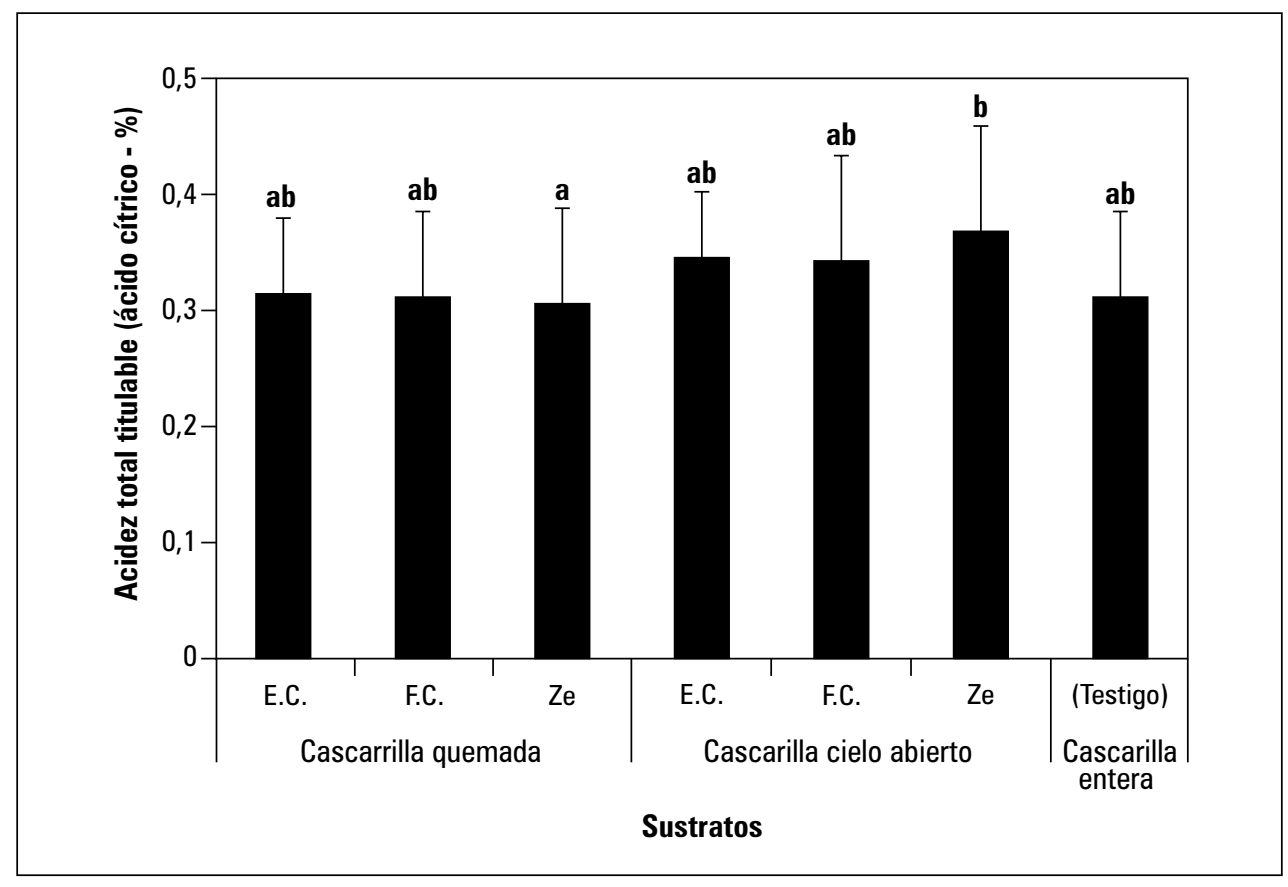

Figura 1. Acidez total titulable expresada como porcentaje de ácido cítrico determinada en frutos de tomate (Solanum lycopersicum L.) cultivados en diferentes mezclas de sustratos. E.C.: escoria de carbón, F.C.: fibra de coco, Ze: zeolita.

fueron aceptables especialmente para la mezcla realizada entre cascarilla cielo abierto y zeolita con el valor más alto comparado con las demás mezclas, ya que estos contenidos de azúcar y de ácido mejoran la calidad de frutos del tomate aunque hay que tener en cuenta la relación entre sólidos solubles totales y la ATT como una expresión de la fruta con un buen equilibrio entre sabores dulces y ácidos (Tzortzak y Economakis, 2008).

\section{Sólidos solubles totales (SST)}

Los resultados obtenidos en esta variable no presentaron diferencias significativas para los factores sustrato y cascarilla. Sin embargo la interacción de sustrato $\times$ cascarilla, mostró diferencias altamente significativas $(P \leq 0,01)$. La mayor variación en los valores de los SST se presentó en la cascarilla quemada (figura 2), en la cual la escoria de carbón aumentó el valor de este parámetro. La escoria de carbón en mezcla con la cascarilla quemada incrementó en $9 \%$ el contenido de SST respecto a la mezcla cascarilla cielo abierto con escoria de carbón. El contenido de SST es un índice importante de transformación. Los SST en este ensayo se ubicaron en un rango entre 4,6 y 5,2 (tabla 3), los cuales se encuentran dentro del rango $(4,59-6,57)$ reportado por Giordano et al. (1994).

En relación con los SST registrados en jugo de frutos, Márquez-Hernández et al. (2006) encontraron que las mejores mezclas para sustratos fueron: vermicomposta al $50 \%$ más arena y vermicomposta con perlita al 25, 37 y $50 \%$, con las cuales se tuvo un impacto positivo en el incremento de los SST. Esto debido probablemente, a que en comparación con la arena probada, esta última tenía baja capacidad de retención de humedad por su porosidad, aunado a una baja capacidad de intercambio catiónico (CIC), caso contrario a lo que ocurrió en las mezclas con compostas. De acuerdo con Heeb et al. (2005), la inclusión de una parte del nitrógeno en forma de nitrato, y de amonio, tiene la capacidad de incrementar el contenido de azúcares y ácidos orgánicos en los frutos, 


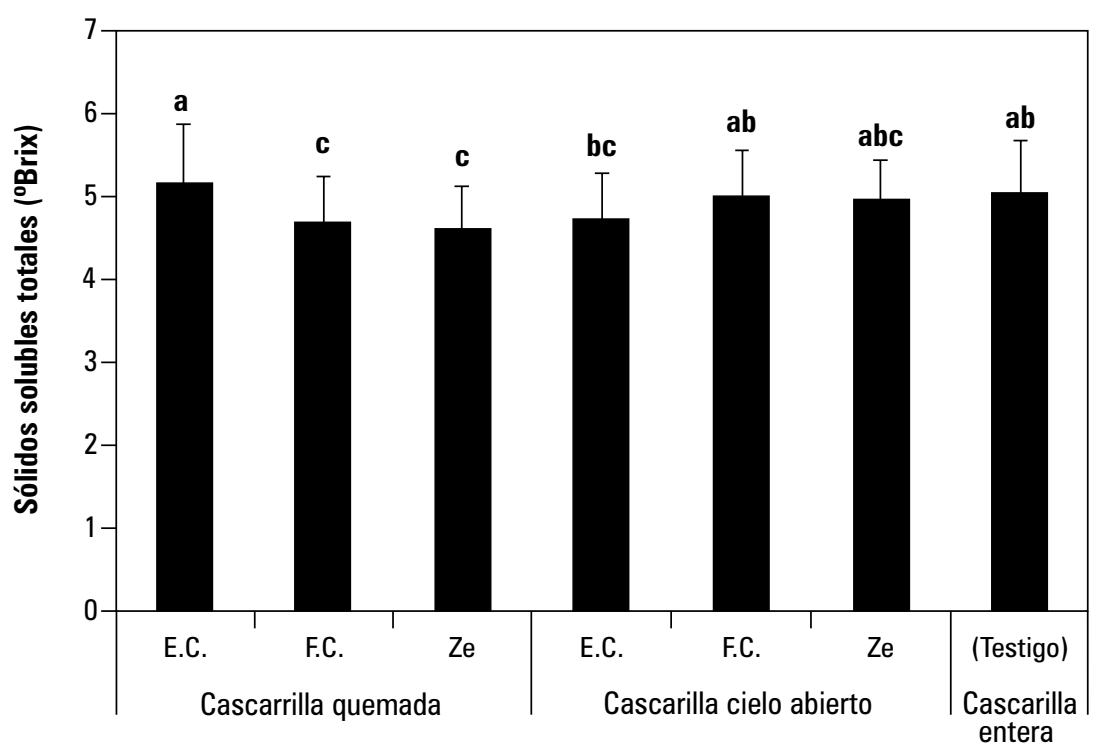

Sustratos

Figura 2. Sólidos solubles totales en frutos de tomate (Solanum lycopersicum L.) cultivados en diferentes mezclas de sustratos. E.C.: escoria de carbón, F.C.: fibra de coco, Ze: zeolita.

en relación con la nutrición exclusivamente con nitrato. De hecho, los sustratos utilizados presentaban diferentes valores en la relación entre amonio y nitratos, lo que pudo afectar este parámetro. Por tanto, las propiedades físicoquímicas de los materiales utilizados en el acondicionamiento de las cascarillas fue determinante en el resultado obtenido en relación con los SST en el presente trabajo.

\section{pH del jugo}

Los resultados hallados en esta variable no arrojaron diferencias estadísticas, sin embargo, los valores del $\mathrm{pH}$ fueron muy cercanos comprendidos entre 4,4 y 4,5 y similares a los reportados por otros investigadores (Pagliarini y Ratti, 1999; Arias et al., 2000; Moraru et al., 2004). Este valor de $\mathrm{pH}$ hace que el tomate sea un producto relativamente fácil de manejar a nivel industrial. Su bajo $\mathrm{pH}$ lo hace poco atractivo a la contaminación microbiana siendo suficiente la pasterización para su envasado tras el proceso de concentrado (Ciruelos et al., 2007). Los mejores valores de $\mathrm{pH}$ para tomates de calidad están entre 4,0 y 5,0 (Aguayo y Artés, 2004), así que los tomates seleccionados para efectos del ensayo se ubican en este rango.

Las cualidades organolépticas del fruto están relacionadas con la composición química. Aguayo y Artés (2004) consideran que para tener un aroma y un sabor óptimos, los tomates deben tener un contenido de sólidos solubles totales entre 4,0 y $6,0^{\circ}$ Brix y un $\mathrm{pH}$ entre 4,0 y 5,0 . Baldwin et al. (1998) consideran que la relación entre los SST y la acidez es un buen indicador para el sabor y el aroma de los tomates.

\section{Peso seco}

Los valores de peso seco encontrados no fueron significativamente diferentes entre cascarillas y sustratos. Más aún, se pudo ver un efecto altamente significativo $(P \leq 0,01)$ de la interacción cascarilla $\times$ sustrato en la materia seca de los frutos evaluados (figura 3). Se observó en la cascarilla cielo abierto la mayor variación para esta variable, cuando se mezcló con zeolita. Es 
de esta manera como la materia seca hallada en tomates cultivados en la mezcla cascarilla cielo abierto con zeolita fue $16,6 \%$ más alta que en la encontrada en la combinación zeolita con cascarilla quemada.

El contenido de materia seca en los frutos de tomate se ubicó entre el 4,5 y 5,4\%. Estos valores se encuentran en el rango para una buena calidad de tomate que está entre un 4,8 a 7,0\% (Winsor, 1976), y fue dependiente de la interacción entre la cascarilla y el sustrato. Estos resultados concuerdan con previos estudios que reportan no encontrar diferencias dependiendo del material sea de origen orgánico o inorgánico sobre peso del fruto de tomate y pepino (Tzortzakis y Economakis, 2008). En el presente estudio, la mezcla realizada entre cascarilla quemada y zeolita no alcanzó el valor mínimo de porcentaje de peso seco en frutos, aunque solo se encuentra $0,03 \%$ por debajo del rango recomendado por Winsor (1976).

\section{Producción de tomate cultivado en las diferen- tes mezclas de sustratos}

La calidad debe definirse en función del uso al que va a ser destinado el producto. En el caso del tomate fresco deben considerarse todas las características valoradas por los consumidores, incluyendo el sabor, el aroma y la textura (Jarén et al., 2006). La calidad de fruto de tomate de acuerdo a los parámetros comerciales mejoró (tabla 3). La producción de tomate de primera calidad, estuvo afectada significativamente $(\mathrm{p} \leq 0,05)$ por el efecto tanto de la cascarilla como del producto mezclado y del mismo modo en la interacción de ambos. De acuerdo con la prueba de Tukey, la cascarilla cruda y la cascarilla cielo abierto fueron estadísticamente iguales, por su parte la cascarilla quemada en promedio presentó el máximo rendimiento para los frutos de primera calidad, dado que las mezclas de sustratos que contenían este material reunían las condiciones adecuadas reportadas por Cadahía (2005),

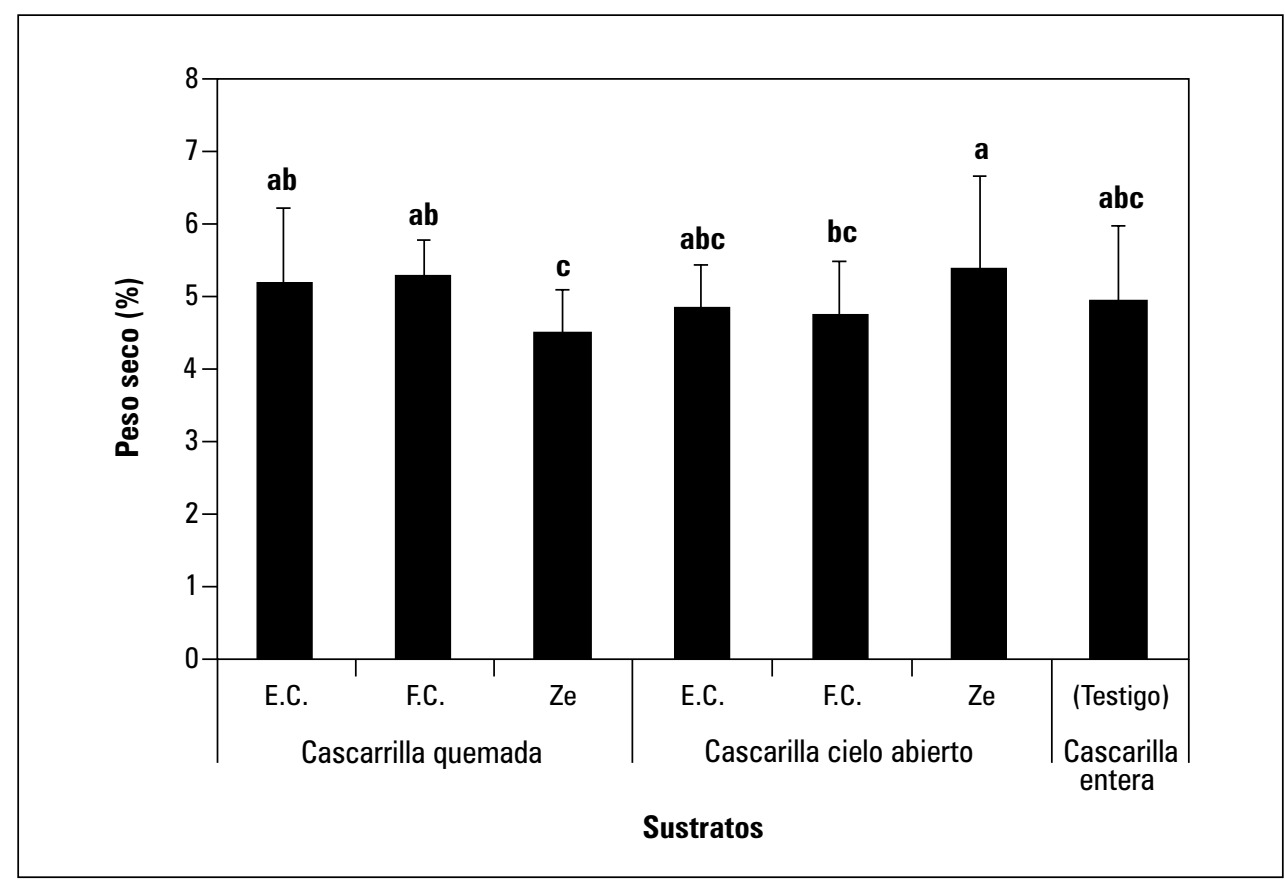

Figura 3. Peso seco en frutos de tomate (Solanum Iycopersicum L.) cultivados en diferentes mezclas de sustratos. E.C.: escoria de carbón, F.C.: fibra de coco, Ze: zeolita. 
quien menciona que un sustrato apropiado para el crecimiento de las plantas, debe tener una alta capacidad de retención de agua, suficiente aireación, baja densidad aparente, alta porosidad total y ser fácilmente mojable. Se observó también el efecto del sustrato en la producción de tomate de esta calidad. En la cascarilla quemada, el mayor promedio se obtuvo con la zeolita, donde se incrementó un 59,6\% la producción comparada con los otros dos sustratos (tabla 3). De igual manera, en la cascarilla cielo abierto, se encontró que la zeolita aumentó en un $57,6 \%$ la producción respecto a los otros dos sustratos (tabla 3). Respecto a la interacción cascarilla x sustrato, se observó que la combinación zeolita con cascarilla quemada incremento la producción de tomate de primera calidad en $22,8 \%$ respecto a la mezcla zeolita con cascarilla cielo abierto.

Para la producción de tomate de calidad industrial estuvo afectada significativamente $(P \leq 0,05)$ tanto por la cascarilla y por el producto mezclado, así como por la interacción de los dos. La producción de tomate de esta calidad en la cascarilla quemada estuvo $27,3 \%$ por encima de la encontrada en la cascarilla cruda. Así mismo, la cascarilla cielo abierto mostró una producción $16,5 \%$ superior a la reportada por la cascarilla cruda (tabla 3). El mayor promedio de producción de esta calidad se encontró en la mezcla de cascarilla quemada con escoria de carbón, la cual fue $14,8 \%$ superior a la observada en la mezcla cascarilla cielo abierto con escoria de carbón. Se debe tener en cuenta que a esta calidad como se había mencionado antes se incluye tomate con cierto grado de malformaciones, leves rayones mecánicos o manchas de poco tamaño, lo cual podría explicar las cantidades superiores en peso presentadas para esta calidad además de afianzar la importancia de un buen manejo fitosanitario y mecánico para la cosecha en el cultivo. Estos resultados pueden explicarse por la mayor capacidad de control de la nutrición y mejor aireación que proporcionan las combinaciones de sustrato evaluadas, con lo que las condiciones de la rizosfera son más favorables que en suelo en todo momento, lo cual redunda en mayor producción y calidad de frutos (Adams, 2002).

\section{Tabla 4. Calidad de fruto medida en función del diámetro ecuatorial del tomate de acuerdo con el peso obtenido en $\mathbf{k g}$.}

\begin{tabular}{|c|c|c|c|c|c|c|}
\hline \multirow{2}{*}{$\begin{array}{c}\text { Tipo de } \\
\text { cascarilla }\end{array}$} & \multirow{2}{*}{ Acondicionador } & \multicolumn{5}{|c|}{ Calidad del fruto $(\mathrm{kg})^{1}$} \\
\hline & & Primera & Segunda & Tercera & Industrial & Desecho \\
\hline \multirow{3}{*}{$\begin{array}{l}\text { Cascarilla } \\
\text { quemada }\end{array}$} & $\begin{array}{l}\text { Escoria de } \\
\text { carbón }\end{array}$ & $5,9 \pm 0,2 b$ & $2,98 \pm 0,4 a$ & $1,6 \pm 0,4 a$ & $19,1 \pm 0,6 b$ & $29,68 \pm 0,4 a$ \\
\hline & Fibra de coco & $5,5 \pm 0,4 a b$ & $6,68 \pm 1,0 b$ & $1,5 \pm 0,2 \mathrm{a}$ & $15,3 \pm 0,6 \mathrm{ab}$ & $29,18 \pm 1,2 \mathrm{a}$ \\
\hline & Zeolita & $13,7 \pm 0,5 d$ & $4,06 \pm 0,6 \mathrm{a}$ & $4,0 \pm 0,7 \mathrm{c}$ & $17,8 \pm 1,4 \mathrm{~b}$ & $39,70 \pm 1,6 \mathrm{a}$ \\
\hline \multirow{3}{*}{$\begin{array}{l}\text { Cascarilla cielo } \\
\text { abierto }\end{array}$} & $\begin{array}{l}\text { Escoria de } \\
\text { carbón }\end{array}$ & $4,7 \pm 0,4 a b$ & $4,30 \pm 0,5 a$ & $1,6 \pm 0,3 a$ & $16,2 \pm 0,25 b$ & $26,94 \pm 1,1 \mathrm{a}$ \\
\hline & Fibra de coco & $4,4 \pm 0,2 \mathrm{a}$ & $3,72 \pm 0,2 \mathrm{a}$ & $3,2 \pm 0,3 b c$ & $16,2 \pm 2,2 \mathrm{ab}$ & $27,66 \pm 1,2 \mathrm{a}$ \\
\hline & Zeolita & $10,6 \pm 0,5 c$ & $4,28 \pm 0,67$ & $2,3 \pm 0,0 a b$ & $12,9 \pm 0,1 \mathrm{a}$ & $30,21 \pm 1,5 \mathrm{a}$ \\
\hline \multicolumn{2}{|c|}{ Cascarilla cruda (control) } & $5,9 \pm 0,3 b$ & $6,10 \pm 0,3 b$ & $3,2 \pm 0,7 b c$ & $12,6 \pm 2,1 \mathrm{a}$ & $27,96 \pm 2,7 \mathrm{a}$ \\
\hline
\end{tabular}

1 Primera: $72-81 \mathrm{~mm}$; Segunda: 62-71 mm; Tercera; $53-61 \mathrm{~mm}$ e Industrial: 6-52 mm. Promedios con letras distintas indican diferencia significativa mínima según la prueba de Tukey $(P \leq 0,05)$. 


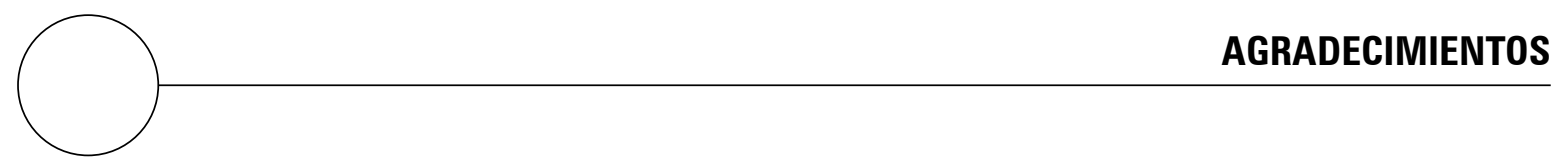

Este estudio fue desarrollado con el apoyo de la Dirección de Investigaciones (DIN) de la Universidad Pedagógica y Tecnológica de Colombia, Tunja, en el marco del plan de trabajo del grupo de investigación Ecofisiología Vegetal, adscrito al programa de Ingeniería Agronómica de la Facultad de Ciencias Agropecuarias.

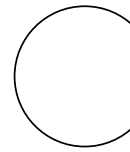

Adams, P. 2002. Nutricional control in hydroponics. En: Savvas, D. y H. Passam (eds.). Hydroponic production of vegetables and ornamentals. Embryo Publ., Atenas. pp. 211-261.

Agronet. 2010. Análisis - Estadísticas. En: http://www. agronet.gov.co; consulta: abril de 2011.

Aguayo, E. y F. Artés, 2004. Elaboración del tomate mínimamente procesado en fresco. Compendios de horticultura 15. Ediciones de Horticultura S.L., Reus, España.

Ansorena, M. y J. 1994. Sustratos: Propiedades y caracterización. Ediciones Mundi-Prensa, Madrid.

Arias, R., T.-C. Lee, D. Specca y H. Janes. 2000. Quality comparison of hydroponic tomatoes (Lycopersicon esculentum) ripened on and off vine. J. Food Sci. 65, 545-548.

Baldwin, E.A., J.W. Scott, T.M.M. Malundo, R.L. Shewfelt y K.S. Tandom. 1998. Relationship between sensory and instrumental analysis for tomato flavor. J. Amer. Soc. Hort. Sci. 123(5), 900-915.

Bastida, T. 2002. Sustratos hidropónicos. Departamento de Preparatoria Agrícola, Universidad Autónoma Chapingo, Chapingo, México.

Cadahía, C. 2005. Fertirrigación: cultivos hortícolas y ornamentales. $3^{\text {a }}$ ed. Ediciones Mundi-Prensa, Madrid.

Calderón, F. y F. Cevallos. 2001. Los sustratos. En: http:// wWw.drcalderonlabs.com/Publicaciones/Los_Sustratos.htm; consulta: 14 de febrero de 2011.

Calderón, F. 2002. Cascarilla de arroz caolinizada. En: http://www.drcalderonlabs.com/Investigaciones/ Cascarilla_Caolinizada/La_Cascarilla_Caolinizada.htm; consulta: 22 de mayo de 2011.

\section{REFERENCIAS BIBLIOGRÁFICAS}

Cánovas, F. 1995. Manejo del cultivo sin suelo. pp. 228254. En: Nuez, F. (ed.). El cultivo del tomate. Ediciones Mundi-Prensa, Madrid.

Ciruelos, A., R. De la Torre y C. González. 2007. Informe 2007. La agricultura y Ganadería Extremeñas Universidad de Extremadura. Parámetros de calidad en el tomate para industria. Pág. 157. En : http://eia.unex.es/EIIAA/Portals/0/La\%20Agricultura\%20y\%201a\%20Ganader\%C3\%ADa\%202007. pdf; consulta : 14 de febrero de 2011.

Djedidi, M., D. Grasopoulos y E. Maloupa. 1997. The effect of different substrates on the quality of Carmello tomatoes (Lycopersicon escullentum Mill.) grown under protection in a hydroponic system. Chaiers Options Mediteraneenes 31, 379-383.

Giordano, I., E. Lahoz, A. D'Amato, A. Pentangelo y A. Sozzi. 1994. Varieties choice of processing tomato in Southern Italy. Acta Hort. 376, 117-122.

Handreck, K. y N. Black. 1991. Particle size analysis and the physical properties of growing media for containers. Comm. Soil Sci. Plant Anal. 14 (3), 209-222

Heeb, A., B. Lundegårdh, T. Ericsson y G.P. Savage. 2005 Nitrogen form affects yield and taste of tomatoes. J. Sci. Food Agr. 85(8), 1405-1414.

Jarén, C., S. Arazuri, M.J. García, P. Arnal y J.I. Arana. 2006. White asparagus harvest date discrimination using NIRS technology. Intl. J. Infrared Millimeter Waves 27(3), 391-401.

Lavado, R. 2000. Aguas y sustratos para la producción ornamental. Origen, propiedades, manejo, influencia sobre los cultivos y determinaciones. New Plant, Buenos Aires, Argentina. 
Maloupa E., I. Mitsios, P.F. Martínez y S. Bladenopoulou. 1992. Study of substrates used in gerbera soilless culture grown in plastic greenhouse. Acta Hort. 323, 139-144.

Márquez-Hernández, C., P. Cano-Ríos, Y.I. Chew-Madinaveitia, A. Moreno-Reséndez y N. RodríguezDimas. 2006. Sustratos en la producción orgánica de tomate cherry bajo invernadero. Rev. Chapingo Ser. Hortic. 12(2), 183-189.

Moraru, C., L. Logendra, T.-C. Lee y H. Janes. 2004. Characteristics of 10 processing tomato cultivars grown hydroponically for the NASA Advanced Life Support (ALS) Program. J. Food Comp. Anal. $17,141-154$.

Pagliarini, E. y S. Ratti. 1999. Relationships between objective measurements and sensory attributes of quality of eight tomato cultivars. Acta Hort. 487, 349-532.

Presidencia de la República de Colombia. 2007. Colombia abre exportaciones de tomate a Costa Rica. En: http://www.presidencia.gov.co/prensa_new/ sne/2007/abril/03/01032007.htm; consulta: abril de 2011 .

Rodríguez, R., J.M. Tabares y J.A. Medina. 2001. Cultivo moderno del tomate. $2^{\mathrm{a}}$. ed. Ediciones Mundi-Prensa, Madrid. pp. 15-23.
Savithri, P., V. Murugappan y R. Nagarajan. 1993. Possibility of economizing $\mathrm{K}$ fertilization by composted coir peat application. Fert. News. 38, 39-40.

Tzortzakis, N. y C. Economakis. 2008. Impacts of the substrate medium on tomato yield and fruit quality in soilless cultivation. Hort. Sci. (Prague) 35(2), 83-89.

Qian, Y.L., A.J. Koski y R. Welton. 2001. Amending sand with isolite and zeolite under saline conditions: leachate composition and salt deposition. HortScience 36, 717-720.

Valdivia, M.A. 1989. Prueba de diferentes sustratos para la producción de jitomate, (Lycopersicon esculentum Mill.) en hidroponía bajo invernadero rústico. Trabajo de grado. Departamento de Fitotecnia, Universidad Autónoma Chapingo, Chapingo, México.

Verdonck, O.F., D. De Vleeschauwer y M. De Boodt. 1981. The influence of the substrate to plant growth. Acta Hort. 126, 251-258.

Wilson, G.C.S. 1985. New perlite system for tomatoes and cucumbers. Acta Hort. 172, 151-156.

Winsor, G.W. 1976. "Tomatoes" appearance to tastequality components. The Grower 45, 33-35. 